\title{
Clinical Pharmacological Considerations on CAR-T Cell Therapy for Cancer
}

\author{
Zhan Wang*1 and Quan Liu ${ }^{2}$ \\ ${ }^{1} \mathrm{MS}$, PhD, Independent Pharmacokinetic and Regulatory Consultant, GoldPkSim LLC, Irvine, CA, USA
}

${ }^{2}$ PhD, Senior Scientist, Stason Pharmaceuticals, Inc., Irvine, CA, USA

Submission: August 02, 2017; Published: August 10, 2017

*Corresponding author: Zhan Wang MS, PhD, Independent Pharmacokinetic and Regulatory Consultant, GoldPkSim LLC, Irvine, CA, Email: GoldPkSim@gmail.com

Keywords: Clinical pharmacology; CAR; CAR-T; Cell therapy; Cancer; Pharmacokinetics, Pharmacodynamics

Abbreviations: ACT: Adoptive Cell Therapy; ADME/ADCE: Absorption, Distribution, Metabolism/Catabolism, and Excretion; AICD: ActivationInduced Cell Death; ALL: Acute Lymphoblastic Leukemia; CAR: Chimeric Antigen Receptor; CD: Cluster of Differentiation; CLL: Chronic Lymphocytic Leukemia; CRS: Cytokine Release Syndrome; FDA: Food and Drug Administration; IL: Interleukin; ITAM: Immunoreceptor Tyrosinebased Activation Motifs; iNKT: invariant Natural Killer T; mAb: monoclonal Antibody; MHC: Major Histocompatibility Complex; NK: Natural Killer; PD: Pharmacodynamics; PK: Pharmacokinetics; scFv: Single-chain variant Fragment; TCR: T Cell Receptor; $\mathrm{T}_{\mathrm{CM}}$ : Central-Memory T; $\mathrm{T}_{\mathrm{EM}}$ : Effector-Memory T; $\mathrm{T}_{\mathrm{ScM}}$ : Stem-Cell Memory T; TLL: Tumor-Infiltrating Lymphocytes

\section{Introduction}

\section{Introduction of CAR-T Cell Therapy}

During the past 70 years, cancer therapy has undergone extensive growth from pharmacotherapy toward targeted therapy, poised upon the considerations of efficacy and safety of clinically applied drugs [1]. The strategy behind this growth was to optimize the structures of the drugs, from synthesized chemical entities to biologics, to enhance the specificity between drugs and disease targets for better potency, and yet to minimize adverse effects to healthy cells. From the 2000s, a better understanding of cancer immunology and an advent of gene transfer technologies provided a possibility to revolutionize the strategy - to modify the therapy-receiver's autologous T cells to fight with his own cancer targets, called chimerical antigen receptor (CAR) T cell therapy [2,3]. This adoptive cell therapy (ACT) is to genetically engineer the collected patients' T-cells to express cell surface receptor(s) (or antibodies, e.g. anti-CD19) specific to cancer targets and subsequently to administer those modified CAR-T cells back into the same patient. Those CAR-T cell react with the cancer targets and start the immune responses that lead to the destruction of cancer cells.

CAR-T therapy recently achieved striking clinical efficacy on B cell-derived hematological malignancies [4-6] and subsequently opened a Pandora box for its multifactorial clinical pharmacology. Clinical pharmacology, usually serves the key to the success of clinical application of drugs by evaluating drugs efficacy and safety. Different from small or large molecules, whose clinical pharmacology can be acquainted through the correlation of well-characterized pharmacokinetics (PK, or ADME/ADCE) with clinical biomarkers or pharmacodynamics (PD), CAR-T cell therapy, a treatment derived from autologous $\mathrm{T}$ cells to the respective patients, encounters challenges to generalize PK and PD knowledge. Yet through CAR-T cells and their binding aspects linking to the efficacy of therapy and safety/tolerability of patients, CAR-T clinical pharmacology could be speculated. This mini-review aims to summarize the general knowledge of CAR-T cell cancer therapy and evaluate the clinical pharmacological considerations through associated PD and PK, mainly based on CAR-T interaction with target.

\section{Pharmacodynamic Considerations upon the Interaction between CAR-T Cells and Cancer Targets}

To know about PD of CAR-T cell therapy, the structure of CAR-T cell and its interaction with cancer targets needs to be 
understood first. Through ex vivo manipulation of patient T cells, CAR-T cells express monomeric receptors usually fused with a spacer element (e.g. the scFv of an anti-cancer mAb that binds to the target), a transmembrane domain, and an intracellular part containing immunoreceptor tyrosine-based activation motifs (ITAMs) [7]. CAR-T cell therapy exploits the normal T cell functions, which enables recognizing and eliminating infected and malignant cells, yet with anticipated higher specificity. Unlike normal $\mathrm{T}$ cell receptor (TCR) undergoing major histocompatibility complex (MHC) dependent binding, which causes the initial phosphorylation of the CD3 zeta-chain within T-cell, CAR-T cells may interact with target epitopes by first recruiting Src kinase to inspire homo-multimerization or interchain phosphorylation of surface chimeric molecules to bind to targets, while concomitantly interacting with endogenous TCR complex with non-covalent ionic bonding [8]. A possible self-protective propensity of CARs - premature exhaustion to tonic signaling and impact efficacy - in the antigen-absence environment during ex vivo culture may be noticed [9].

To obtain a specific and sustained interaction between CAR-T cells and cancer targets for anti-cancer effects, despite the natural antigen-expression levels of targets, CAR-T cells' overall affinity to the targets and the accessibility to epitopes are the two factors to be evaluated. Efforts to modify those factors were embraced, for example, by increasing the spacer flexibility to reach poorly accessible epitopes. Specific designs on spacers can also be used to stimulate activation-induced cell death (AICD) in xenograft mouse models [10]. Enhanced efficacy and minimized toxicity could therefore be achieved by optimizing the combination of those factors for each patient [11]. Besides $\mathrm{T}$ lymphocytes that most CAR development focuses on, other effectors, such as natural killer (NK) cells, invariant NKT (iNKT) cells, different subtype of T cells, were undergone investigation to become promising cell types for CAR redirection [12]. Therefore, within PD domain, tuning of CAR affinity to improve potency and mitigate potential toxicity toward the normal cells, enhancing spacer element engagement to increase epitope accessibility, and even identifying novel effectors cell types may be considered.

\section{Clinical Pharmacokinetic Considerations of CAR-T Cells}

The conventional ADME criteria are likely not applicable here for CAR-T cells. Yet clinical pharmacokinetic considerations of CAR-T cells can be also evaluated based on the interaction between CAR-T and cancer targets. The interactions described previously focused on the situations when CAR-T cells have already migrated to the cancer target microenvironment or adhere to the endothelial surface of the cancer cells [13]. CAR-T cells' ability to migrate into the cancer environment is also a key factor relevant to efficacy. Enhancement of the specificity to bind to the target is likely a key relevant to migration. Further biodistribution of administered CAR-T cells relies on a process of chemokine-driven extravasation into antigen-rich tissues (e.g. solid tumor), so called infiltration [11]. During infiltration, the important process associated with efficacy, CAR-T cells may encounter T-cell defense associated with degraded heparanase [14] and aforementioned immunogenicity due to the transient up-regulation of exhaustion markers, leading to poor infiltration. A resolving method was to genetically complementing CAR-T cells with heparanase. Better penetration into solid tumor mass was then observed.

In addition to wide biodistribution, the long term in vivo exposure of CAR-T is a critical and superior propensity compared to pharmacotherapy or even targeted therapy, as low frequent (at least once every month) clinical dosing regimens can be planned and with enhanced anti-cancer effect. To elongate CAR-T in vivo exposure, the costimulatory endodomains approach was investigated. It was revealed that anti-CD19 CAR-T cells incorporating 4-1BB endodomains, compared to CD28 endodomains, had extended the in vivo (mouse model) exposure of CAR-T cells for months [15]. However, like anti-drug antibody to biologics, the anti-transgene immunogenicity rising from the immune-rejection of the host to the different species that CAR-T cells are derived from can shorten the exposure to less than one week. Therefore, pre-treatment of immunosuppression leading to lymphodepletion of the resident $\mathrm{T}$ cells provided a strategy to sensitize the host to CAR-T cells and subsequently achieve longterm in vivo exposure. Nowadays, the $2^{\text {nd }}$ or $3^{\text {rd }}$ generation of CAR-T cells by incorporating 1 or 2 costimulatory endodomains, respectively, to down-grade immunogenicity and optimize in vivo exposure may also be considered [16].

The in vivo exposure of CAR-T cells is also determined by their memory-differentiation phenotype state after modification before administered into patients. Four states were differentiated in sequence from naïve $\mathrm{T}$ cells, throughout $\mathrm{T}_{\mathrm{SCM}}$ and $\mathrm{T}_{\mathrm{CM}}$, finally to $\mathrm{T}_{\mathrm{EM}}$. Interestingly, the in vivo exposure inversely correlates with the $\mathrm{T}$ cell memory-differentiation phenotype, such as $\mathrm{T}$ cells in the $\mathrm{T}_{C M}$ state displayed longer in vivo exposure than in the $\mathrm{T}_{\mathrm{EM}}$ state, likely due to inability of $\mathrm{T}_{\mathrm{EM}}$ to produce IL-2, a cytokine for homeostasis [16]. Thus, maintaining CAR-T cells at a preterminally differentiated state could also improve their in vivo exposure.

\section{Toxicity of CAR-T Cell Therapy}

During the clinical development, usually before proofof-concept of targeted efficacy, safety/tolerability on healthy subjects or patients is the first thing to be considered. After CAR-T cell therapy, the commonly reported toxicities in CAR-T cell therapy are the cytokine release syndrome (CRS) and normally in concomitance with neurotoxicity $[11,16]$. The former arises from the intensive release of cytokines, such as TNF-alpha, IL- 6 and IFN-gamma, with clinical signs of fever, hypotension, tachycardia and respiratory distress. Those intense inflammations occurred soon after the therapy and reached toward a peak within 
days. The latter usually occurs accompanying with CRS, with signs of confusion, seizures, aphasia, hallucinations, delirium. Fortunately, those neurological manifestations appear to be transient and reversible without long-term deficits yet with unknown mechanisms. Compared to those unfatal toxicities, persistent B-cell aplasia was more profound and increased the risk of infection which may lead to hypogammaglobulinemia, while the other one time on-target off-tumor toxicity caused fatal incidence [17]. Currently, there is no clinical or regulatory standard to guide the toxicity management, while prophylaxis, medicine and supportive care were used in the practice.

\section{Future Prospects}

CAR-T cell therapy becomes an emerging area to treat cancers, in particular for hematological malignancies. With the rising of pharmaceutical startups focusing on CAR-T cell therapy, such as Kite Pharma and Juno Therapeutics, Inc., and efforts from the existing big pharma as well as the need to maturate the criteria to guide clinical development by regulatory agencies, the next decade will be a critical period to expand the science in this field. Surprisingly, the sprout of this (as part of ACT) therapy took place in 1980s [18]; its evolution in the past 30 years from pioneering using lymphokine-activated killer cells, through different immune effectors, such as tumorinfiltrating lymphocytes (TIL) [19], to TCR and CAR, paved the recent impressive successes in refractory B-ALL and promising responses in B-cell NHL and CLL [4]. Although the clinical application and practice of CAR-T cell therapy remains in fancy, the recent FDA advisory committee's recommendation for approval of the $1^{\text {st }}$ CAR-T cell therapy, Novartis' tisagenlecleucel for pediatric or young adult B-cell ALL, demonstrated a large foot step on the road toward defeating cancer as well as promoting personalized/precision medicine. Future availability of advanced technologies and revelation of the unknown areas of human and cancer immunology will continue to improve the safety and efficacy in CAR-T cell therapy, for example, by producing new generation CAR-T cells (e.g. bi-specifics) [20]. Regarding spreading the success to non-hematological (not B-lineage restricted) malignancies, challenges such as identifying good targets to maintain the specificity and enhance infiltration, are expected to be concurred.

\section{References}

1. Chabner BA, Roberts TG Jr. (2005) Timeline: chemotherapy and the war on cancer. Nat Rev Cancer 5(1): 65-72.

2. Kantarjian H, Sawyers C, Hochhaus A, Guilhot F, Schiffer C, et al. (2002) Hematologic and cytogenetic responses to imatinib mesylate in chronic myelogenous leukemia. N Engl J Med 346(9): 645-652.

3. Gill S, June CH (2015) Going viral: chimeric antigen receptor T-cell therapy for hematological malignancies. Immunol Rev 263(1): 68-89.

4. Turtle CJ (2014) Chimeric antigen receptor modified T cell therapy for B cell malignancies. Int J Hematol 99(2): 132-140.
5. Turtle CJ, Maloney DG (2016) Clinical trials of CD19-targeted CARmodified T cell therapy; a complex and varied landscape. Expert Rev Hematol 9(8): 719-721.

6. Davila ML, Riviere I, Wang X, Bartido S, Park J, et al. (2014) Efficacy and toxicity management of 19-28z CAR T cell therapy in B cell acute lymphoblastic leukemia. Sci Transl Med 6(224): 224ra25.

7. Eshhar Z, Waks T, Gross G, Schindler DG (1993) Specific activation and targeting of cytotoxic lymphocytes through chimeric single chains consisting of antibody-binding domains and the gamma or zeta subunits of the immunoglobulin and T-cell receptors. Proc Natl Acad Sci USA 90(2): 720-724.

8. Bridgeman JS, Ladell K, Sheard VE, Miners K, Hawkins RE, et al. (2014) $\mathrm{CD} 3 \zeta$-based chimeric antigen receptors mediate $\mathrm{T}$ cell activation via cis- and trans-signalling mechanisms: implications for optimization of receptor structure for adoptive cell therapy. Clin Exp Immunol 175(2): 258-267.

9. Long AH, Haso WM, Shern JF, Wanhainen KM, Murgai M, et al. (2015) 4-1BB costimulation ameliorates $\mathrm{T}$ cell exhaustion induced by tonic signaling of chimeric antigen receptors. Nat Med 21(6): 581-590.

10. Berger C, Sommermeyer D, Hudecek M, Berger M, Balakrishnan A, et al. (2015) Safety of targeting ROR1 in primates with chimeric antigen receptor-modified T cells. Cancer Immunol Res 3(2): 206-216.

11. Norelli M, Casucci M, Bonini C, Bondanza A (2016) Clinical pharmacology of CAR-T cells: Linking cellular pharmacodynamics to pharmacokinetics and antitumor effects. Biochim Biophys Acta 1865(1): 90-100.

12. Metelitsa LS, Wu HW, Wang H, Yang Y, Warsi Z, et al. (2004) Natural killer $\mathrm{T}$ cells infiltrate neuroblastomas expressing the chemokine CCL2. J Exp Med 199(9): 1213-1221.

13. Ahmed N, Brawley VS, Hegde M, Robertson C, Ghazi A, et al. (2015) Human epidermal growth factor receptor2 (HER2)-specific chimeric antigen receptor-modified $\mathrm{T}$ cells for the immunotherapy of HER2positive sarcoma. J Clin Oncol 33(15): 1688-1696.

14. Caruana I, Savoldo B, Hoyos V, Weber G, Liu H, et al. (2015) Heparanase promotes tumor infiltration andantitumor activity of CAR-redirected $\mathrm{T}$ lymphocytes. Nat Med 21(5): 524-529.

15. Maude SL, Frey N, Shaw PA, Aplenc R, Barrett DM, et al. (2014) Chimeric antigen receptor $\mathrm{T}$ cells for sustained remissions in leukemia. $\mathrm{N}$ Engl J Med 371(16): 1507-1517.

16. Davila ML, Sadelain M (2016) Biology and clinical application of CAR T cells for B cell malignancies. Int J Hematol 104(1): 6-17.

17. Morgan RA, Yang JC, Kitano M, Dudley ME, Laurencot CM, Rosenberg SA (2010) Case report of a serious adverse event following the administration of $\mathrm{T}$ cells transduced with a chimeric antigen receptor recognizing ERBB2. Mol Ther 18(4): 843-851.

18. Rosenberg SA, Lotze MT, Muul LM, Leitman S, Chang AE, et al. (1985) Observations on the systemic administration of autologous lymphokine-activated killer cells and recombinant interleukin-2 to patients with metastatic cancer, N Engl J Med 313(23): 1485-1492.

19. Bondanza A, Valtolina V, Magnani Z, Ponzoni M, Fleischhauer K, et al. (2006) Suicide gene therapy of graft-versushost disease induced by central memory human T lymphocytes. Blood 107(5): 1828-1836.

20. Dudley ME, Wunderlich JR, Robbins PF, Yang JC, Hwu P, et al. (2002) Cancer regression and autoimmunity in patients after clonal repopulation with antitumor lymphocytes. Science 298(5594): 850854. 
This work is licensed under Creative Commons Attribution 4.0 License

DOI: 10.19080/JPCR.2017.03.555619
Your next submission with Juniper Publishers will reach you the below assets

- Quality Editorial service

- Swift Peer Review

- Reprints availability

- E-prints Service

- Manuscript Podcast for convenient understanding

- Global attainment for your research

- Manuscript accessibility in different formats ( Pdf, E-pub, Full Text, Audio)

- Unceasing customer service

Track the below URL for one-step submission https://juniperpublishers.com/online-submission.php 